\title{
Leveraging social networks for anytime-anyplace health information
}

\author{
Scott H. Burton • Kesler W. Tanner • \\ Christophe G. Giraud-Carrier
}

Received: 18 April 2012/Revised: 10 July 2012/ Accepted: 17 July 2012

(C) Springer-Verlag 2012

\begin{abstract}
The health landscape is shifting to one in which common individuals are no longer merely consumers, but also producers, of health information. We demonstrate that social media platforms provide the means to seek and receive personalized, credible health advice from peers at any place and time, by tracking dental health advice sought and received in Twitter. We show that for genuine dental advice-seeking questions, answers are received $32 \%$ of the time, with the first reply coming less than 6 min after the question is posed, in the median. We compare our results to studies focusing on generic questions and find stronger relationships between users that answer health questions. Additionally, we find that users with more social capital, in the form of more reciprocal follower/following relationships, are more likely to receive responses and receive them faster, and are thus better able to leverage their social networks in receiving advice.
\end{abstract}

Keywords Social Q\&A · Social capital · Twitter · Health advice

\section{Introduction}

Historically, individuals have been regarded as patients, or mere consumers of health information and care, provided directly, and controllably, by experts including medical

\footnotetext{
S. H. Burton $(\bowtie) \cdot$ K. W. Tanner · C. G. Giraud-Carrier Brigham Young University, Provo, UT 84602, USA e-mail: sburton@byu.edu

K. W. Tanner

e-mail: keslert@gmail.com

C. G. Giraud-Carrier

e-mail: cgc@cs.byu.edu
}

doctors and other health practitioners. The advent of the Internet and the proliferation of online content of all kinds, including health-related content, means that more information is now readily accessible by lay individuals, thus enabling health information consumption on a much larger scale, and independently of the traditional channels of distribution (e.g., doctor's office, hospitals). Furthermore, individuals are not limited to simply looking up information; they are also able to use such technology as blogs and online forums to discuss, comment on, and share experiences about various health issues they themselves, or their loved ones, may be facing. In doing so, individuals begin to change slowly from being only consumers of health information to becoming producers of the same. What they share with others about symptoms, side-effects, remedies and other relevant experiential knowledge becomes information for others to consume. We regard this as the first phase of the transformation of the health care landscape.

The second phase, and the one really responsible for a major shift in attitude and behavior, is the emergence of rich, interactive social media applications, such as Facebook, MySpace, YouTube or Twitter. These applications allow individuals to connect, collaborate, and exchange their current thoughts, feelings and activities with one another without concern for geographical boundaries. What that means is that whereas the role of providing support and advice regarding health issues has generally been limited to health care providers one-on-one visits, and to close associations in one's family or small network of friends, it may now be extended to all participants in one's social network. Hence, we are witnessing a dramatic paradigm shift in health care. The one-way flow of health information and solutions from health care professionals who produce them to lay individuals who consume them is gradually being replaced by a more fluid and distributed flow where any 
and all individuals, professionals or otherwise, may act as both consumers and producers of health information, advice and solutions. This new state of affairs is of course not without its own challenges, including privacy, quality and trust issues. We do not address these here, however, but focus instead on showing how social media are indeed being used to seek and receive health advice.

A 2008 survey indicates that a large number of people turn to the Internet (59\%) and social media (34\%) for health information (Elkin 2008). The fact that turning to search engines is often the first action people take for health questions is precisely what enables identifying early-stage outbreaks through search query tracking (Brownstein et al. 2010; Ginsberg et al. 2008; Wilson and Brownstein 2009; Pelat et al. 2009). When it comes to personal health, there are, however, some limitations to the Internet:

1. One has to search for the needed information and possibly wade through many results.

2. The information tends to be of a generic nature and hence responses are not personalized.

3. The information available is limited to what authors have already posted, which may or may not include what one is looking for.

4. The credibility of the information is a concern as inaccuracies can arise from under-informed people sharing opinions, as well as businesses and other invested parties promoting their own agendas or manipulating the content to their own ends (Freeman and Chapman 2007; Moturu et al. 2008; Hossler and Conroy 2008; Deloitte Center for Health Solutions 2010a; Deloitte Center for Health Solutions 2010b).

Some level of context and credibility (or trust) can be established through focused social media groups (Greene et al. 2011) and stand-alone e-communities such as Patients Like $\mathrm{Me},{ }^{1}$ which provide opportunities for people with common conditions to connect. However, despite sharing common conditions, users of these forums often have little history outside these interactions. The blogosphere is another rich source of health data (Neustein 2007; Miller and Pole 2010; West et al. 2011), where users are sometimes familiar with blog authors, either personally or through consistent online interaction and following. So while unknown bloggers carry the same risks to validity as other Internet sites, trusted authors can provide a sense of integrity. However, even respected authors and sites can only be probed for existing information, and not questioned in real-time for advice.

$\overline{{ }^{1} \mathrm{http} / / / \mathrm{www}}$.patientslikeme.com.
Asking questions in social networks provides a natural mechanism to overcome all of the above limitations, since it exhibits the following characteristics.

1. No search. One need not search for answers but simply ask questions to his or her network and wait for answers to come.

2. Personalization. One is more likely to receive personalized answers because such answers are to a specific question and come from people one knows.

3. Versatility. One may obtain information about almost any topic, including some that are not easily obtained through search engines (Morris et al. 2010a).

4. Credibility. Answers and advice received from one's network are more credible, or carry a higher level of trust, since social network connections are based on established relationships (either in the real or in the virtual world).

Furthermore, social media provide the additional advantage that questions may be asked at any time, which is desirable since health needs and questions arise in many different settings, often outside of the doctor's office or hospital. And responses may also be received at any time, often shortly after the question has been asked (Timeliness), as we shall see.

In this paper, we demonstrate the value of social media for health advice seeking, as discussed above, by showing how people use Twitter to ask and receive advice about dental health issues. Twitter is particularly attractive for this type of study for a number of reasons. First, it is a very rich source of timely, spontaneous, and uncensored excerpts of users' emotions and activities. It is estimated that over 200 million tweets are generated each day. Second, Twitter implements a one-to-many broadcast communication mechanism in which a user may pose a question to all of his or her followers at once. And finally, Twitter possesses a rich application programming interface (API) that allows information to be filtered and/or searched programmatically.

The contribution of this work is twofold. First, we establish that despite possible concerns of anonymity or privacy, social media users are seeking advice, and receiving responses, in at least some areas of health (in our case, demonstrated by dental advice in Twitter). Second, we highlight social factors that contribute to speed and quantity of responses.

The remainder of the paper is organized as follows. First, we discuss related work and then outline our methodology for finding dental advice-seeking questions and their responses on Twitter. Next, we present our results followed by a discussion of the implications of our findings and the differences of our results compared to general question and answer research. Finally, we offer conclusions and suggest areas for future work. 


\section{Related work}

Social network analysis is becoming increasingly important in health and bioinformatics as relationships are modeled between people, cancer cells (Bortolussi and Policriti 2012), or even between related diseases and genes (Xiang et al. 2012). The impact of online social networks on public and personal health is increasingly being recognized (Kamel Boulos and Wheelert 2007; Chiu et al. 2009; Vance et al. 2009; Ma et al. 2010; Eysenbach 2009; Savage 2011). Several recent studies have specifically identified health topics in Twitter data. Scanfeld et al. (2010) mined Twitter content and demonstrated that social media provide a means for sharing health information, especially as it relates to antibiotics misuse and understanding. Paul and Dredze (2011) employed topic modeling to 1.5 million tweets and were able to discover that numerous healthrelated conditions (e.g., allergies, obesity and insomnia) were mentioned in the tweets. Prier et al. (2011) were able to identify tobacco-related conversations through Twitter. Chew and others conducted content analyses of tweets on H1N1 and swine flu mentions and demonstrated the value of using the tool for monitoring pandemics (Chew and Eysenbach 2010; Lampos and Cristianini 2010; Aramaki et al. 2011). Finally, in the area of dental health, Heaivilin et al. (2011) characterized tweets relating to dental pain. We build upon their work by considering dental advice being sought, as opposed to merely statements of pain, and also identify the answers received to the dental questions.

Ma et al. (2010) have shown that online social interactions may carry enough positive peer pressure to encourage healthy behavior. It has also been found that, while in some cases anonymity may promote increased antagonism (Lange 2007), adolescents generally feel more comfortable discussing potentially embarrassing topics with some degree of anonymity, as afforded by chat rooms and bulletin boards (Gould et al. 2002; Suzuki and Calzo 2004). While these studies have shown the effectiveness of several Internet tools, such as bulletin boards and chat rooms, to our knowledge, health advice seeking has not been studied in social media platforms, such as Twitter, which introduce a different dynamic of at least partially surrendered anonymity because of explicit connections to either real- or virtual-world friends.

Advice seeking presupposes the formulation of question to be asked of one's social network. Identifying questions is a non-trivial process, especially in micro-text posts where space limitations discourage proper grammar and promote abbreviations and slang, which produce challenges for traditional natural language processing techniques such as part-of-speech tagging (Dent and Paul 2011). Because of the difficulty of directly applying NLP techniques, to find questions for study and analysis, other approaches have been taken. Morris et al. (2010a, b) were the first ones to study the use of social media for asking questions. In their work, however, they do not analyze media content directly, but rely instead on survey techniques where a number of individuals were asked about their experience in asking questions, receiving responses and providing responses themselves on Twitter or Facebook. Efron and Winget (2010) look at tweets directly and employ a keyword approach. We adopt a keyword-based approach focused specifically on finding advice-seeking questions, as opposed to the more general topic of all interrogative statements. In that sense, we are influenced by the work of Paul et al. (2011a, b), who analyzed questions and answers found in Twitter based on the presence of a question mark, and then used Amazon's Mechanical Turk to restrict the candidate set to valid questions, as judged by the turkers. We follow a similar approach, where we first identify likely advice-seeking questions related to dental health, and post-process them through human readers to increase precision.

\section{Methods}

To illustrate the value of social media in seeking and receiving health advice, we focus on dental health issues in Twitter. The topic of dental work is of general interest, because all people must manage their dental health to some degree. It also provides an area that people are generally comfortable discussing and where the vocabulary is accessible to common individuals. The common vocabulary of dental health, and the fact that complex medical terminology is typically not used, helps in identifying dental advice, and better enables a keyword-based approach.

Because the Twitter platform limits tweets to 140 characters, it may inherently promote questions and responses that are less complex or elaborate. On the other hand, the simplistic nature of tweets may also cause more directly asked questions and more succinct responses. The direct nature of tweets is helpful to our study, wherein we are seeking to determine if health advice is being sought and obtained.

We received an exemption from the university Internal Review Board to study these public-facing tweets.

\subsection{Observing dental tweets}

The first step to identify dental advice is obtaining a sample of tweets on the dental topic. Twitter provides a streaming API that returns a portion of the complete stream of tweets filtered by a search query. To identify potential dental tweets, we filtered the Twitter stream by the keywords: 
"tooth," “teeth," “dental," “dentist," “gums," “molar," "moler," "floss," and "toothache." This keyword-based filter does not guarantee that all resulting tweets are related to dental health. For example, tweets containing the words "sweet tooth" or "molar mass" will pass through our filter even though they clearly have nothing to do with dental issues. However, this simple mechanism provides a good starting point.

Using our filter, we observed all tweets for two separate weeks, from October 26 to November 1, 2011, and from November 9 to November 15, 2011, and received a total of 1,032,754 tweets over the 14-day period, for an average of approximately 74,000 tweets per day.

\subsection{Identifying advice-seeking questions}

Twitter essentially implements a broadcast, one-to-many, form of communication in which a user posts messages (status messages, reactions to current events, questions, etc.), generally intended to be read by all of that user's followers. This is an ideal mechanism for soliciting advice, because the question can be posed once to multiple potential respondents, as opposed to, for example, making individual phone calls to friends. Probably due in part to this broadcast-style of communication, we have observed that many advice-seeking tweets tend to contain words such as "anyone" or "anybody" with a question mark at the end of the sentence (e.g., "anyone know of a good dentist in Lancaster?", "Cold and sore throat has developed into painful tooth/mouth ache. This one's totally new to me. Can anyone enlighten me?").

Interestingly, Morris et al. (2010b) who characterized questions on Twitter, found that, in their set, $81.5 \%$ of questions contained question marks, and $20.9 \%$ contained the word "anyone." In their work on characterizing questions on Twitter, Paul et al. (2011a) simply used the question mark to identify questions, which allowed them to find more questions, many of which, however, were rhetorical. Because we are not concerned with categorizing all questions, but rather, are focused on genuine, health advice-seeking questions, we have found the use of the additional anyone/anybody criterion to help in removing some of the rhetorical, sarcastic, and advertising questions.

From the roughly one million potential dental tweets, looking for the words "anybody," "anyone," or "any1," together with a question mark, we identified 2,035 candidate dental advice-seeking questions. To further improve the precision of our set of questions, we followed an approach similar to Paul et al. (2011b), except that, since we had several available, we used willing volunteers rather than Amazon's Mechanical Turk. In all, we had 18 independent individuals read the candidate tweets and manually classify them. Each person classified approximately 200 tweets, according to the following criterion:

\section{Mark the tweet as a health advice-seeking question if it seems clear that the individual posting the tweet is asking for advice about a dental health issue regarding themselves or their family, with the expectation of receiving a response.}

The condition about "themselves or their family" allowed us to eliminate generic questions and questions about pets, while the condition about "expectation of receiving a response" helped us focus on questions most likely to seek timely advice. Each tweet was independently classified by two different people. The separate classifications were in agreement in $87 \%$ of cases, and the remaining tweets were arbitrated by the authors. Of the 2,035 candidate questions, $432(21 \%)$ were labeled as dental advice-seeking questions, such as: "does anyone know how long it takes for swelling on your mouth to go down after getting teeth out?" and "Can anyone suggest some home remedies for a \#toothache?". Many of the tweets not matching the above criterion were in fact valid questions but did not seek dental advice or did not seem to be expecting an actual response (e.g., "Going to the dentist this morning. Anyone want to trade? I'll even throw in my best marble!", "anyone know how do to the putty for vampire teeth?" (sic.)). ${ }^{2}$

\subsection{Identifying responses}

One of the shortcomings of the Twitter API is that it does not allow direct querying of responses to a particular tweet. To overcome this limitation, we used the search API to identify any tweets after the question was issued that were directed to the author of the question, using the @username syntax. Then, using the detailed REST API, we examined each of these possible replies individually to determine if it was listed as being "in-reply-to" the original question, as specified by a meta-data field of the tweet. While it is possible that users might respond by simply creating a new tweet addressed to the author, we assume that most users actually make use of the "reply" feature of the Twitter website (also available in most popular third-party applications), which ensures that the reply-to meta-data field is correctly populated. Because of this assumption we may overlook some replies, causing some of our results to be underestimates, but we can have high confidence that the responses we identify are truly replies to the original question.

\footnotetext{
2 Note that one of our weeks of study included the Halloween holiday, which resulted in several questions about costume elements such as vampire teeth.
} 
Table 1 Tweets at each stage of the experiment

\begin{tabular}{lrl}
\hline Set & Tweets & $\begin{array}{l}\text { Percent of } \\
\text { previous set (\%) }\end{array}$ \\
\hline Matching dental keywords & $1,032,754$ & \\
Candidate advice-seeking questions & 2,035 & 0.2 \\
$\begin{array}{l}\text { Quality advice-seeking questions } \\
\text { (human verified) }\end{array}$ & 432 & 21.2 \\
Questions that received answers & 140 & 32.4 \\
\hline
\end{tabular}

Because Twitter is asynchronous, there are potentially many different strands of conversation occurring simultaneously. This means that a user may pose dental questions to their followers but then continue to converse with others about different topics and receive a response later. However, the longer it has been since a question was asked, the less likely it is to receive a response. Paul et al. (2011a), for example, observed that $67 \%$ of their responses came within $30 \mathrm{~min}$ and $95 \%$ came within $10 \mathrm{~h}$. Because it is possible that health-advice replies may take longer than replies to other questions, but still recognizing that they become less likely over time, and less relevant, we searched for those occurring within $48 \mathrm{~h}$ of the original question tweet.

We applied this process of determining dental replies to our 432 dental advice-seeking questions, and found that $140(32 \%)$ received at least one response. In the median case, the first response was received $5.5 \mathrm{~min}$ after the question was asked. As noted, because our approach focused on minimizing the number of false positives, we cannot deduce that the other questions, for which we did not identify a response, were truly left unanswered. The number of tweets at each stage of the experiment is shown in Table 1.

\section{Results}

Because Timeliness is one of the desirable properties of social media, we feel it is useful to determine the time of day and week when questions were asked. To do so, we converted the question tweet's time to the user's local time wherever a time zone was listed on the user account. Week days are defined as Monday-Friday from 8 a.m. to 5 p.m. Week nights are defined as Monday-Thursday from 5 p.m. until the next day at 8 a.m. Weekends are defined as Friday at 5 p.m. until Monday at 8 a.m. Finally, "any after hours" is a combination of week nights and weekends. The results are summarized in Table 2 and Fig. 1.

As shown in Table 2, the majority of the dental adviceseeking questions were posted during the evening and
Table 2 Distribution of questions and responses by time of day and week

\begin{tabular}{lllll}
\hline & Total & $\begin{array}{l}\text { Receiving } \\
\text { replies }\end{array}$ & $\begin{array}{l}\text { Without } \\
\text { replies }\end{array}$ & $\begin{array}{l}\text { Reply } \\
\text { percent }\end{array}$ \\
\hline Week day & 123 & 48 & 75 & 39.0 \\
Week night & 105 & 44 & 61 & 41.9 \\
Week end & 72 & 21 & 51 & 28.4 \\
Any after hours & 177 & 65 & 112 & 36.3 \\
No time zone & 132 & 27 & 105 & 20.5 \\
\hline
\end{tabular}

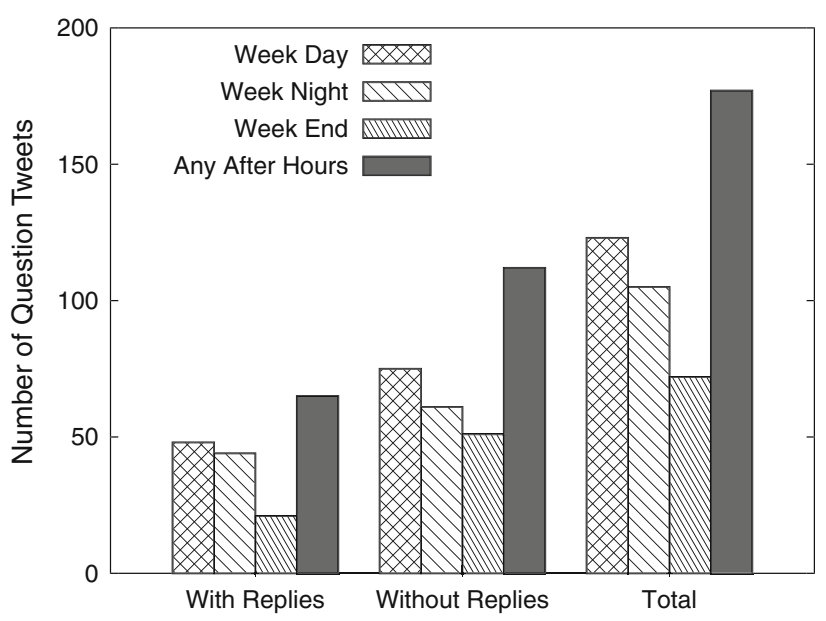

Fig. 1 Number of questions occurring during week days, week nights, and week ends. "Any after hours" includes both week nights and weekends, and represents the majority of the questions

weekend hours, which is not necessarily surprising given that we observed that Twitter activity is highest in the evening in general. However, this may be particularly relevant in the context of dental advice because it represents advice sought when traditional channels, namely dentist offices, are not available. It is interesting to note that over $36 \%$ of the questions asked after hours received answers, with slightly more of the week nights questions being answered (42\%) than the weekend questions ( $28 \%$ ). The latter could be explained by the fact that users may be less apt to consume others' content on the weekends, possibly catching up on their feeds on Monday morning, thus responses, even if they were to be given, would likely appear beyond our $48 \mathrm{~h}$ limit, at which stage they would also have become much less useful as more traditional channels would have re-opened.

While we cannot measure Credibility directly, we do, as others have (e.g., see Paul et al. 2011a), look at the influence that an individual's, e.g. network (i.e., its followers and its followings) may have on the responses they receive. As shown in Table 3, those users who received replies had 
Table 3 Advice-seeking questions receiving replies and relationship to, e.g. network

\begin{tabular}{lllll}
\hline & Total & Percent & $\begin{array}{l}\text { Median } \\
\text { followers }\end{array}$ & $\begin{array}{l}\text { Median } \\
\text { following }\end{array}$ \\
\hline Receiving replies & 140 & 32.4 & 331.5 & 256.5 \\
Without replies & 292 & 67.6 & 136.0 & 157.0 \\
\hline
\end{tabular}

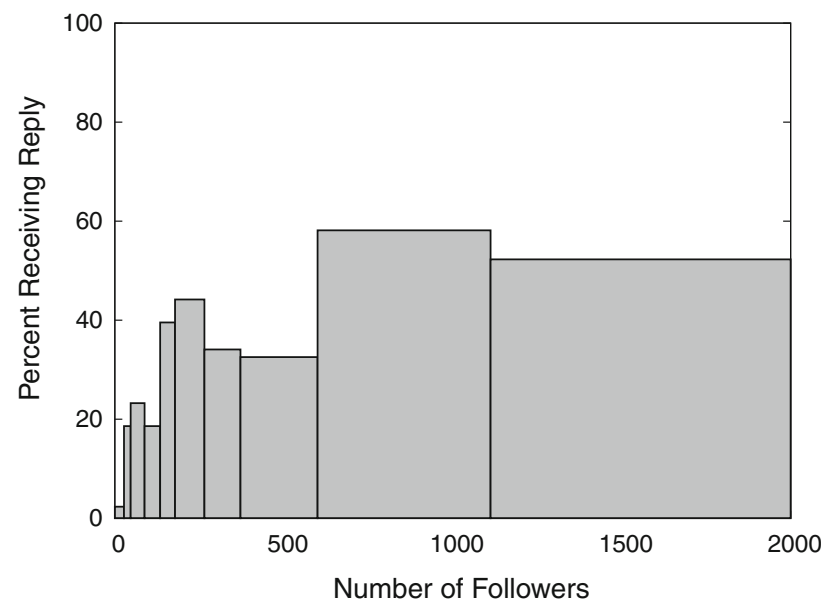

Fig. 2 The percent of questions receiving replies based on the number of followers, grouped into 10 bins of equal-question frequency

significantly (unpaired, two-tailed $t$ test, $p=0.005$ ) more followers (median of 331.5) than those that did not receive replies (median of 136). This is further demonstrated in Fig. 2 which shows the percent of questions receiving answers based on the number of followers, grouped into 10 bins of equal frequency with regard to the number of questions. While on average $32 \%$ of questions received replies, users that had more than 200 followers had their questions answered $45 \%$ of the time, and users will less than 100 followers received answers in only $14 \%$ of cases.

Additionally, as shown in Figs. 3 and 4, those users with more followers received their first replies faster on average. For questions that received replies, Fig. 3 shows the delay between the question and the first response based on the number of followers, and Fig. 4 shows the same data, grouping the questions into 10 bins of equal frequency with respect to the number of questions. For questions that received answers, the number of replies also correlates positively with the number of followers (Pearson's $r=0.48$ ), suggesting that users with more followers are not only more likely to receive responses but also more likely to receive more of them.

In addition to considering followers and following independently, it is interesting to look at the reciprocity of

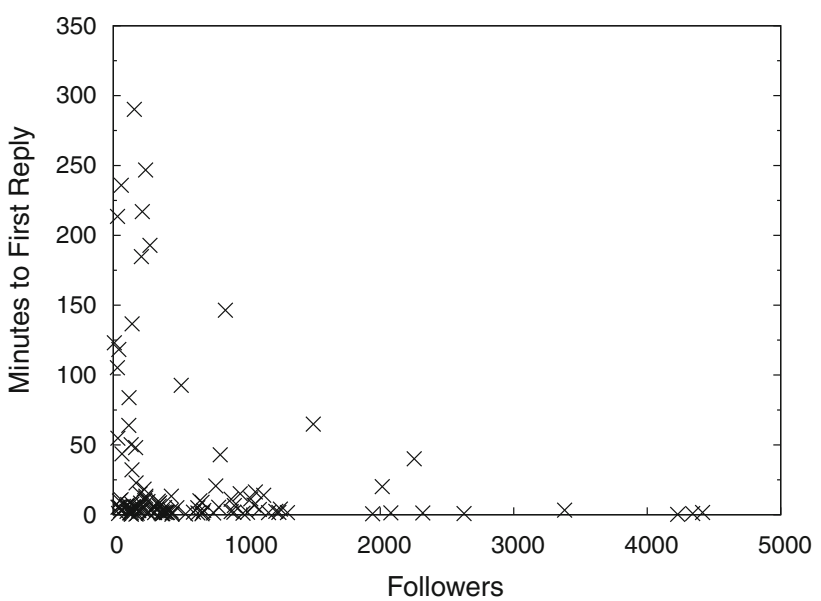

Fig. 3 Time taken to receive the first reply versus number of followers

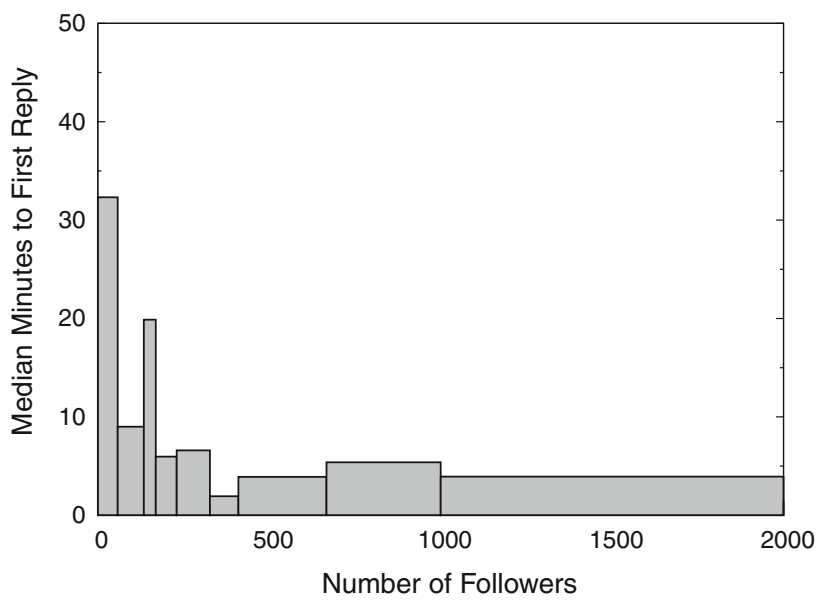

Fig. 4 Median number of minutes to the first reply by number of followers, grouped into 10 bins of equal-question frequency

Table 4 Reciprocity of relationships between askers and responders

\begin{tabular}{lcc}
\hline Relationship & Amount & Percent \\
\hline No relation & 16 & 6.6 \\
Responder following asker & 226 & 93.0 \\
Asker following responder & 170 & 70.0 \\
Mutual following and follower & 169 & 69.5 \\
\hline
\end{tabular}

the relationship between the asker and responder, as this provides a better indication of the strength of the relationship between the two. As shown in Table 4, $93 \%$ of responses came from users following the person asking the question and $69.5 \%$ came from users with a mutual following/follower relationship. 
Table 5 Coarse approximations of asker's view of social capital value

\begin{tabular}{lll}
\hline Relationship & Value & Reason \\
\hline $\begin{array}{l}\text { No relationship } \\
\text { Followed by } \\
\text { responder }\end{array}$ & Low & $\begin{array}{l}\text { Users may not know each other } \\
\text { Asker may not know responder }\end{array}$ \\
$\begin{array}{c}\text { Following } \\
\text { responder }\end{array}$ & Med-high & $\begin{array}{c}\text { Asker trusts responder but responder } \\
\text { may not know asker well }\end{array}$ \\
$\begin{array}{c}\text { Mutual } \\
\text { relationship }\end{array}$ & High & $\begin{array}{c}\text { Asker trusts responder who can give } \\
\text { personalized advice }\end{array}$ \\
\hline
\end{tabular}

\section{Discussion}

Our finding that, overall, $32 \%$ of advice-seeking questions received answers is significantly higher than the rate of $9 \%$ that Paul et al. (2011a) observed for personal and health-related questions. This may be due to a number of factors, such as dental topics being less sensitive or personal than other health topics, but most likely it is the result of our focus on questions where a response was actually expected, rather than including rhetorical ones. The fact that users that are genuinely seeking advice receive it $32 \%$ of the time suggests that Twitter is a valid resource to turn to for personalized answers. And, seeing that users with more than 200 followers received answers to $45 \%$ of their questions and those with less than 100 followers only received answers to $14 \%$ of questions demonstrates that users with more social capital are better able to leverage their network to receive value-in this case, health advice.

The implicit social capital graph among Twitter users implies a weighting of different connections, where a person values their relationship with others at very different levels, ranging from very little weight with unknown users to a strong connection with close personal friends or family members. While the actual weighting of the graph would be very fine-grained, the following/follower structure of Twitter could provide a coarse approximation where low value exists between users that are not following or followed by one another, and high value exists in mutual follower/following relationships. Possible coarse approximations for social capital values from the asker's point of view are summarized in Table 5 .

The fact that having more followers results in a greater likelihood of receiving a response as well as more timely responses is not necessarily surprising. Statistically speaking, the simple fact that more people are likely to view the message means that it is more likely to be seen, and seen sooner. However, this result also says something about the Personalization available in social media. Indeed, people generally invest a lot of time and energy into building their social networks. In the case of Twitter, this means following other users, as well as responding to questions and posting relevant status updates regularly in an attempt to gain followers. In doing so, users create social capital and maintain a list of followers who come to know them. The more social capital a user has, the better his or her chances of getting timely responses, and of obtaining responses that are more personalized.

Furthermore, since $69.5 \%$ of the answers came from responders who had a mutual relationship with the asker (both following and followed by), we may be able to argue that the responses are not only more personalized but also more credible. This number $(69.5 \%)$ of reciprocal relationships is significantly higher than the $36 \%$ found by Paul et al. (2011a). The difference may be due to the fact that giving health advice is more personal than answering other questions. Thus, the mere act of answering a health question may indicate a strong relationship between the two users. In any case, these results suggest that success in obtaining advice on social media may be directly related to an individual's social capital. Others have similarly suggested that answering the questions of others could be used as a means to increase one's social capital thus resulting in higher chances of having one's own questions answered (Morris et al. 2010b).

The short delay to answer is rather remarkable. In the median case, the first response was received within $5.5 \mathrm{~min}$ of the question being asked. Given that many responses were given outside of normal office hours, this suggests that Twitter may be effective at handling non life-threatening health emergencies.

\section{Conclusions and future work}

Social media offer unique opportunities for people seeking health advice in that information may be obtained in a more timely manner, on a potentially broader set of issues than present in other media (e.g., Internet), with increased credibility and better personalization. We have used Twitter and dental health issues as an example to demonstrate that (1) people do ask dental health-related questions on Twitter, (2) a large number of questions are answered, (3) users receive timely advice after business hours thus making social media a valuable addition to traditional channels, and (4) the pattern of connections between askers and responders suggests that social capital is a determinant factor in the process.

The fact that advice can be obtained from established relationships, in particular mutual follower/following connections, provides an increased level of personalization and trust over anonymous Internet forum posts. And the fact that users with higher social capital are better able to leverage their networks for health advice demonstrates the value in building and maintaining on-line social relationships. 
There are several interesting areas of future work. First, we have done nothing here to test the validity of the responses received, but have assumed that since they came from "trusted" sources, they too could be trusted. It would be interesting to test this hypothesis formally, perhaps involving subject matter experts to evaluate the actual quality (and safety) of the health advice offered. Second, while we obtain promising results with dental issues, we would need to repeat our study with other health topics to see whether the results generalize or whether there are any differences across health topics, possibly due to the sensitivity of the topic. Finally, we have discussed social capital and argued that there was evidence that social capital had a direct impact on one's ability to obtain answers to advice-seeking questions. Again, this result deserves more analysis. Furthermore, it would be interesting to expand the study of the role of social capital on Twitter by checking whether people are indeed more likely to turn to Twitter (or some other social media) than to a less personal medium, such as the Internet, to get answers to their question. Also, recognizing that social networks are dynamic, it would be valuable to study (and potentially predict) how the network might change as a result of asking or answering questions, especially recognizing that links could be both added and dropped as a result of this interaction (Almansoori et al. 2012).

\section{References}

Almansoori W, Gao S, Jarada T, Elsheikh A, Murshed A, Jida J, Alhajj R, Rokne J (2012) Link prediction and classification in social networks and its application in healthcare and systems biology. Network Model Anal Health Inf Bioinf 1:27-36

Aramaki E, Maskawa S, Morita M (2011) Twitter catches the flu: detecting influenza epidemics using twitter. In: Proceedings of the 2011 conference on empirical methods in natural language processing (EMNLP2011), pp 1568-1576

Bortolussi L, Policriti A (2012) Studying cancer-cell populations by programmable models of networks. Network Model Anal Health Inf Bioinf. doi:10.1007/s13721-012-0010-x

Brownstein JS, Freifeld CC, Chan EH, Keller M, Sonricker AL, Mekaru SR, Buckeridge DL (2010) Information technology and global surveillance of cases of $2009 \mathrm{~h} 1 \mathrm{n} 1$ influenza. N Engl J Med 362(18):1731-1735

Chew C, Eysenbach G (2010) Pandemics in the age of twitter: content analysis of tweets during the 2009 h1n1 outbreak. PLoS One 5(11):e14-e118

Chiu D, Ande P, Coward R, Woywodt A (2009) The times they are a changin: the internet and how it affects daily practice in nephrology. NDT Plus 2(4):273

Deloitte Center for Health Solutions (2010a) 2010 survey of health care consumers. http://www.deloitte.com/assets/Dcom-United States/LocalAssets/Documents/US $\_C H S \backslash \_2010 S u r v e y o f H e a l t h$ CareConsumers $\_$050310.pdf (accessed Jan 2012)

Deloitte Center for Health Solutions (2010b) Social networks in health care: communication, collaboration and insights. http://www. deloitte.com/assets/Dcom-UnitedStates/LocalAssets/Documents/ US $\_C H S \backslash 2010$ SocialNetworks $\_070710 . p d f$
Dent K, Paul S (2011) Through the twitter glass: Detecting questions in micro-text. In: Proceedings of the AAAI 2011 workshop on analyzing microtext, pp 8-13

Efron M, Winget M (2010) Questions are content: a taxonomy of questions in a microblogging environment. Proc Am Soc Inf Sci Technol 47(1):1-10

Elkin N (2008) How America searches: health and wellness. http:// www.icrossing.com/sites/default/files/how-america-searcheshealth-and\%-wellness.pdf (accessed Jan 2012)

Eysenbach G (2009) Infodemiology and infoveillance: framework for an emerging set of public health informatics methods to analyze search, communication and publication behavior on the internet. J Med Internet Res 11(1):e11

Freeman B, Chapman S (2007) Is "YouTube" telling or selling you something? Tobacco content on the YouTube video-sharing website. Tobacco Control 16(3):207

Ginsberg J, Mohebbi M, Patel R, Brammer L, Smolinski M, Brilliant L (2008) Detecting influenza epidemics using search engine query data. Nature 457(7232):1012-1014

Gould M, Munfakh J, Lubell K, Kleinman M, Parker S (2002) Seeking help from the internet during adolescence. J Am Acad Child Adolesc Psychiatry 41(10):1182-1189

Greene J, Choudhry N, Kilabuk E, Shrank W (2011) Online social networking by patients with diabetes: a qualitative evaluation of communication with Facebook. J Gen Intern Med 26(3):287-292

Heaivilin N, Gerbert B, Page J, Gibbs J (2011) Public health surveillance of dental pain via twitter. J Dent Res 90(9):1047-1051

Hossler E, Conroy M (2008) YouTube as a source of information on tanning bed use. Arch Dermatol 144(10):1395-1396

Kamel Boulos M, Wheelert S (2007) The emerging web 2.0 social software: an enabling suite of sociable technologies in health and health care education. Health Inf. Libr J 24(1):2-23

Lampos V, Cristianini N (2010) Tracking the flu pandemic by monitoring the social web. In: 2nd International workshop on cognitive information processing (CIP), IEEE, pp 411-416

Lange $P$ (2007) Commenting on comments: investigating responses to antagonism on YouTube. In: Proceedings of the 70th annual conference of the Society for Applied Anthropology, p 2007

Ma X, Chen G, Xiao J (2010) Analysis of an online health social network. In: Proceedings of the 1st ACM international health informatics symposium, pp 297-306

Miller E, Pole A (2010) Diagnosis blog: checking up on health blogs in the blogosphere. Am J Publ Health 100(8):1514-1519

Morris M, Teevan J, Panovich K (2010a) A comparison of information seeking using search engines and social networks. In: Proceedings of the 4th international AAAI conference on weblogs and social media, pp 291-294

Morris MR, Teevan J, Panovich K (2010b) What do people ask their social networks, and why? A survey study of status message q\&a behavior. In: Proceedings of the 28th international conference on human factors in computing systems. ACM, New York, pp 1739-1748

Moturu S, Liu H, Johnson W (2008) Trust evaluation in health information on the world wide web. In: 30th annual international conference of the IEEE engineering in Medicine and Biology Society (EMBS), IEEE, pp 1525-1528

Neustein A (2007) Sequence package analysis: a new natural language understanding method for intelligent mining of recordings of doctor-patient interviews and health-related blogs. In: Fourth international conference on information technology (ITNG), IEEE, pp 431-438

Paul M, Dredze M (2011) You are what you tweet: analyzing Twitter for public health. In: International AAAI conference on weblogs and social media (ICWSM)

Paul S, Hong L, Chi E (2011a) Is Twitter a good place for asking questions? A characterization study. In: Proceedings of the 5th 
international AAAI conference on weblogs and social media, pp 578-581

Paul S, Hong L, Chi E (2011b) What is a question? Crowdsourcing tweet categorization. In: Proceedings of the CHI 2011 workshop on crowdsourcing and human computation

Pelat C, Turbelin C, Bar-Hen A, Flahault A, Valleron A (2009) More diseases tracked by using Google trends. Emerg Infect Dis 15(8): 1327

Prier KW, Smith MS, Giraud-Carrier C, Hanson CL (2011) Identifying health-related topics on twitter: an exploration of tobaccorelated tweets as a test topic. In: Proceedings of the 4th international conference on social computing, behavioralcultural modeling and prediction, Springer, Berlin, pp 18-25

Savage N (2011) Twitter as medium and message. Commun ACM 54(3): $18-20$

Scanfeld D, Scanfeld V, Larson E (2010) Dissemination of health information through social networks: Twitter and antibiotics. Am J Infect Control 38(3):182-188
Suzuki L, Calzo J (2004) The search for peer advice in cyberspace: an examination of online teen bulletin boards about health and sexuality. J Appl Dev Psychol 25(6):685-698

Vance K, Howe W, Dellavalle R (2009) Social internet sites as a source of public health information. Dermatol Clin 27(2):133-136

West J, Hall P, Hanson C, Thackeray R, Barnes M, Neiger B, McIntyre E (2011) Breastfeeding and blogging: exploring the utility of blogs to promote breastfeeding. Am J Health Educ 42(2):106-115

Wilson K, Brownstein J (2009) Early detection of disease outbreaks using the internet. Canad Med Assoc J 180(8):829

Xiang Y, Fuhry D, Kaya K, Jin R, Çatalyürek Ü, Huang K (2012) Merging network patterns: a general framework to summarize biomedical network data. Netw Model Anal Health Inf Bioinf. doi:10.1007/s13721-012-0009-3 